\title{
Virtual Partitioning for Connection Admission Control in Cellular/WLAN Interworking
}

\author{
Enrique Stevens-Navarro and Vincent W.S. Wong \\ Department of Electrical and Computer Engineering \\ The University of British Columbia, Vancouver, Canada \\ E-mail: \{enriques, vincentw\}@ece.ubc.ca
}

\begin{abstract}
Wireless wide area networks (WWANs) and wireless local area networks (WLANs) have complementary characteristics which make them suitable to jointly offer an ubiquitous wireless solution. In cellular/WLAN interworking, the quality of service $(\mathrm{Q} o S)$ requirements for different services (e.g., voice and real-time video) can be guaranteed by using connection admission control. In this paper, we propose the use of virtual partitioning (VP) [5] resource sharing scheme to facilitate admission control in a multi-service integrated cellular/WLAN system. VP preallocates a nominal capacity for each service based on the expected traffic and the required blocking probabilities. We first determine the policy functions corresponding to VP for new and handoff connection requests. Then, three different nominal capacities for VP are compared with the cutoff priority policy. Numerical results show that lower blocking and dropping probabilities can be achieved by VP in a wide range of conditions.
\end{abstract}

\section{INTRODUCTION}

It is envisioned that wireless wide area networks (WWANs), such as third generation $(3 \mathrm{G})$ wireless cellular systems, can be integrated with wireless local area networks (WLANs) to offer ubiquitous Internet access and IP multimedia services to mobile users. Various interworking architectures have been proposed to integrate both networks [1]. The IEEE has set up the 802.21 working group to standardize inter-operability between 802 and non-802 networks (e.g., 3G cellular) [2]. The cellular standardization bodies such as the $3 \mathrm{G}$ Partnership Project (3GPP) and 3GPP2 are extending the packet data and IP multimedia services to the WLAN environment [3], [4].

In an integrated cellular/WLAN system, WLANs are deployed in densely populated areas to provide local coverage, while cellular networks are used to provide wide area coverage. Users carrying mobile devices equipped with dual interfaces can establish connections with different access networks. As they move within the coverage areas, they are able to switch connections among networks. This process is called handoff or handover. It is called horizontal if it is between two networks which use the same access technology (e.g., between two WLANs, or between two neighboring cells in a cellular network). On the other hand, it is called vertical if it is between two networks which use different access technologies (e.g., from a cell to a WLAN, or vice versa).

Connection admission control can guarantee the quality of service (QoS) of different IP multimedia applications (e.g., voice, real-time video) by limiting the number of connections admitted into a network. An admission control policy can either accept the connection request and allocate the resources accordingly, or reject (i.e., block/drop) the connection request. Usually, higher priority is given to the requests from the handoff users, as opposed to the requests from the new users.

Virtual Partitioning (VP) was proposed in [5] for robust resource sharing in multi-service wired networks. VP allows the sharing of network resources among several traffic classes in an efficient, fair, and robust manner. It works under the idea of pre-allocating a nominal capacity for each service based on the expected traffic and required QoS in terms of blocking probabilities. In [6], VP was used for admission control in wireless cellular networks. Several models with preemption are evaluated in terms of new and handoff blocking probabilities, systems utilization, and packet loss probability.

We now describe some of the related work on admission control and resource management in cellular/WLAN interworking aiming to differentiate service requirements. In [7], the WLAN-first admission control scheme is proposed where both voice and data connection requests within the overlapped coverage area are sent to the WLAN. In [8], a randomized guard channel admission control policy is used in which a random number of channels is reserved for voice handoffs. Recently, in [9], service-based handoff schemes in which connection requests from vertical handoff users can be placed into a queue are investigated. In [10], a multi-service common radio resource management scheme is presented. It proposed a network selection algorithm for connection requests from the new users, and a handoff algorithm for requests from the handoff users. In [11], joint radio resource management and spectrum auction algorithms combining technical and economic aspects are used to improve the usage of the networks' radio resources. In [12], we proposed a multiservice cellular/WLAN interworking model and investigated the combinations of two admission control polices. By using policy functions, we extended the cutoff priority $(\mathrm{CP})$ from [13], and the fractional guard channel (FG) from [14] as admission control policies in an integrated system. The policy functions are defined based on the service type (e.g., voice, data), the type of connection request (i.e., new or handoff), and the admission control policy being used.

In this paper, we propose the use of VP for connection admission control in cellular/WLAN interworking. We implement VP following the concept of policy functions proposed in [12]. The contributions of our work are as follows: 
- VP is extended for connection admission control in the integrated cellular/WLAN system, and its corresponding policy functions are determined.

- The performance of the system is evaluated by using VP with three different nominal allocations, and compared with the CP admission policy. Results show that VP can provide lower blocking and dropping probabilities in a wide range of conditions.

The rest of the paper is organized as follows. The model for the integrated cellular/WLAN system is summarized in Section II. Policy functions for VP are formulated in Section III. Numerical results are presented in Section IV. Conclusions and future work are given in Section V.

\section{BACKGROUND AND PREVIOUS WORK}

We use the integrated cellular/WLAN system proposed in [12]. For the sake of clarity and completeness, we introduce the notations and summarize the relevant parts in this section.

Let $M^{c}$ denote the set of cells of a cellular network, and $W_{i}^{c}$ denote the set of WLANs inside the coverage area of cell $i$ of the cellular network. Let $S$ denote the set of all multimedia services. Each service $s \in S$ requires $b_{s}$ basic bandwidth units (BBUs) or channels to guarantee its QoS requirements. The new connection requests for service $s$ arrive at cell $i$ and WLAN $k$ according to independent Poisson processes with rates $\lambda_{i_{s}}^{c}$ and $\lambda_{k_{s}}^{w}$, respectively. The channel holding times for service $s$ at cell $i$ and WLAN $k$ are exponentially distributed with parameters $\mu_{i_{s}}^{c}$ and $\mu_{k_{s}}^{w}$, respectively. The channel occupancy of every cell $i$ for all $i \in M^{c}$ and WLAN $k$ for all $k \in W_{i}^{c}$ evolves according to an independent multidimensional birth-death process [15].

A birth event happens when a connection request from a handoff or a new user is accepted. A death event happens when a user terminates its connection or leaves a cell or a WLAN. The multi-dimensional birth-death process has $|S|$ dimensions, where $|\cdot|$ denotes the set's cardinality. The $s^{t h}$ dimension models the channel occupancy evolvement due to the changes in the number of connections of service $s$.

Let define $\boldsymbol{m}_{\boldsymbol{i}}^{\boldsymbol{c}}=\left(m_{i_{1}}^{c}, m_{i_{2}}^{c}, \ldots, m_{i_{S}}^{c}\right)$ for all $i \in M^{c}$, and $\boldsymbol{m}_{\boldsymbol{k}}^{\boldsymbol{w}}=\left(m_{k_{1}}^{w}, m_{k_{2}}^{w}, \ldots, m_{k_{S}}^{w}\right)$ for all $k \in W_{i}^{c}$ as the channel occupancy vectors in cell $i$ and WLAN $k$, respectively. Each vector indicates the number of active connections of each service $s$. A channel occupancy vector $\boldsymbol{m}_{\boldsymbol{i}}^{\boldsymbol{c}}$ is feasible if $m_{i_{s}}^{c} \geq 0$ for all $s \in S$ and $\sum_{s \in S} m_{i_{s}}^{c} b_{s} \leq C_{i}^{c}$ holds, where $C_{i}^{c}$ is the capacity of cell $i$ in BBUs. We denote the set of all feasible $\boldsymbol{m}_{\boldsymbol{i}}^{\boldsymbol{c}}$ as $\Theta_{i}^{c}$. Similarly, a channel occupancy vector $\boldsymbol{m}_{\boldsymbol{k}}^{\boldsymbol{w}}$ is feasible if $m_{k_{s}}^{w} \geq 0$ for all $s \in S$ and $\sum_{s \in S} m_{k_{s}}^{w} b_{s} \leq C_{k}^{w}$ holds, where $C_{k}^{w}$ is the capacity of WLAN $k$ in BBUs. We denote the set of feasible $\boldsymbol{m}_{\boldsymbol{k}}^{\boldsymbol{w}}$ as $\Theta_{k}^{w}$.

Horizontal and vertical handoff rates for each service $s$ are defined between each cell and WLAN as in [12]. The handoff rates define a set of fixed-point equations that can be solved iteratively by repeated substitutions [16]. With the birth and death rates (which depend on the handoff rates), we are able to calculate $P_{i}^{c}\left(\boldsymbol{m}_{\boldsymbol{i}}^{\boldsymbol{c}}\right)$ and $P_{k}^{w}\left(\boldsymbol{m}_{\boldsymbol{k}}^{\boldsymbol{w}}\right)$, which are the probabilities of being in states $\left(m_{i_{1}}^{c}, m_{i_{2}}^{c}, \ldots, m_{i_{S}}^{c}\right)$ and $\left(m_{k_{1}}^{w}, m_{k_{2}}^{w}, \ldots, m_{k_{S}}^{w}\right)$ in the $|S|$-dimensional birth-death processes corresponding to cell $i$ and WLAN $k$, respectively.

Let $B_{n_{i_{s}}}^{c}$ and $B_{h_{i_{s}}}^{c}$ denote the probability of blocking and dropping a connection request for service $s$ in cell $i$ for new and handoff users, respectively. We have,

$$
\begin{aligned}
B_{n_{i_{s}}}^{c} & =\sum_{\boldsymbol{m}_{\boldsymbol{i}}^{\boldsymbol{c}} \in \Theta_{i}^{c}} P_{i}^{c}\left(\boldsymbol{m}_{\boldsymbol{i}}^{\boldsymbol{c}}\right) \beta_{n_{i_{s}}}^{c}\left(\boldsymbol{m}_{\boldsymbol{i}}^{\boldsymbol{c}}\right), \\
B_{h_{i_{s}}}^{c} & =\sum_{\boldsymbol{m}_{\boldsymbol{i}}^{\boldsymbol{c}} \in \Theta_{i}^{c}} P_{i}^{c}\left(\boldsymbol{m}_{\boldsymbol{i}}^{\boldsymbol{c}}\right) \beta_{h_{i_{s}}}^{c}\left(\boldsymbol{m}_{\boldsymbol{i}}^{\boldsymbol{c}}\right),
\end{aligned}
$$

where $\beta_{n_{i_{s}}}^{c}\left(\boldsymbol{m}_{\boldsymbol{i}}^{\boldsymbol{c}}\right)$ and $\beta_{h_{i_{s}}}^{c}\left(\boldsymbol{m}_{\boldsymbol{i}}^{\boldsymbol{c}}\right)$ are the policy functions for connection requests for service $s$ in cell $i$ from new and handoff users, respectively.

The policy functions can evaluate the performance of different admission control policies in an integrated cellular/WLAN system. The policy function $\beta_{n_{i_{s}}}^{c}\left(\boldsymbol{m}_{\boldsymbol{i}}^{\boldsymbol{c}}\right)$ determines the probability of not accepting connection requests from new users for service $s$ in cell $i$. Similarly, $\beta_{h_{i s}}^{c}\left(\boldsymbol{m}_{\boldsymbol{i}}^{\boldsymbol{c}}\right)$ determines the probability of not accepting connection requests from handoff users for service $s$ in cell $i$. Since the connection requests from the handoff users have higher priority than the requests from the new users, it usually assumed that $\beta_{h_{i_{s}}}^{c}\left(\boldsymbol{m}_{\boldsymbol{i}}^{\boldsymbol{c}}\right) \leq \beta_{n_{i_{s}}}^{c}\left(\boldsymbol{m}_{\boldsymbol{i}}^{\boldsymbol{c}}\right)$ for all $i \in M^{c}$ and $s \in S$.

Let $B_{n_{k_{s}}}^{w}$ and $B_{h_{k_{s}}}^{w}$ denote the probability of blocking and dropping a connection requests for service $s$ in WLAN $k$ for new and handoff users, respectively. We have,

$$
\begin{aligned}
B_{n_{k_{s}}}^{w} & =\sum_{\boldsymbol{m}_{\boldsymbol{k}}^{\boldsymbol{w}} \in \Theta_{k}^{w}} P_{k}^{w}\left(\boldsymbol{m}_{\boldsymbol{k}}^{\boldsymbol{w}}\right) \beta_{n_{k_{s}}}^{w}\left(\boldsymbol{m}_{\boldsymbol{k}}^{\boldsymbol{w}}\right), \\
B_{h_{k_{s}}}^{w} & =\sum_{\boldsymbol{m}_{\boldsymbol{k}}^{\boldsymbol{w}} \in \Theta_{k}^{w}} P_{k}^{w}\left(\boldsymbol{m}_{\boldsymbol{k}}^{\boldsymbol{w}}\right) \beta_{h_{k_{s}}}^{w}\left(\boldsymbol{m}_{\boldsymbol{k}}^{\boldsymbol{w}}\right),
\end{aligned}
$$

where $\beta_{n_{k_{s}}}^{w}\left(\boldsymbol{m}_{\boldsymbol{k}}^{\boldsymbol{w}}\right)$ and $\beta_{h_{k_{s}}}^{w}\left(\boldsymbol{m}_{\boldsymbol{k}}^{\boldsymbol{w}}\right)$ are the policy functions for connection requests for service $s$ in WLAN $k$ from new and handoff users, respectively. The policy functions model the behavior of the admission control policies, and determine the probability of not accepting a connection for each type of request, and for each type of service, given the channel occupancy vectors $\boldsymbol{m}_{\boldsymbol{i}}^{\boldsymbol{c}}$ and $\boldsymbol{m}_{\boldsymbol{k}}^{\boldsymbol{w}}$.

By using policy functions, in [12], we extended two admission control policies: CP from [13] and FG from [14]. Admission control policy $\mathrm{CP}$ reserves a fixed number of channels exclusively for connection requests from handoff users. Then, for CP policy a connection request from a new user of service $s$ in cell $i$ is accepted if $\sum_{s \in S} m_{i_{s}}^{c} b_{s} \leq T_{i_{s}}^{c}$, and rejected otherwise. Parameter $T_{i_{s}}^{c}$ is used to tune the threshold to give priority for requests from handoff users. Note that for all $i \in M^{c}$ and $s \in S$, we have: $0 \leq T_{i_{s}}^{c} \leq C_{i}^{c}-b_{s}$. A similar parameter $T_{k_{s}}^{w}$ is used for CP policy to set the priority of the handoff connection requests over the new requests in WLAN $k$ for all $k \in W_{i}^{c}$ and $s \in S$.

In this paper, we extend VP for admission control in cellular/WLAN interworking using policy functions. 


\section{ViRTUAL PARTITIONING FOR ADMISSION CONTROL IN CELLULAR/WLAN INTERWORKING}

\section{A. Admission Control Policy}

To recapitulate, we consider an integrated cellular/WLAN system consisting of $\left|M^{c}\right|$ cells with $\left|W_{i}^{c}\right|$ WLANs inside each cell. The capacity of cell $i$ is $C_{i}^{c}$ BBUs for all $i \in M^{c}$, and the capacity of WLAN $k$ is $C_{k}^{w}$ BBUs for all $k \in W_{i}^{c}$. There are $|S|$ multimedia services, each requiring $b_{s}$ BBUs or channels to satisfy its QoS requirements. VP stipulates for each service $s$, a nominal capacity or nominal allocation in each cell $i$ and WLAN $k$, according to:

$$
\begin{aligned}
& \sum_{s \in S} C_{s}^{c} \geq C_{i}^{c}, \quad \forall i \in M^{c}, \\
& \sum_{s \in S} C_{s}^{w} \geq C_{k}^{w}, \quad \forall k \in W_{i}^{c},
\end{aligned}
$$

where $C_{s}^{c}$ and $C_{s}^{w}$ are the nominal capacities in BBUs for service $s$ in the cellular network and in the WLANs, respectively.

The idea of VP, as introduced in [5], is that if a service is using less than its nominal allocation, it is considered to be underloaded. On the other hand, if a service is using more than its nominal allocation, it is considered to be overloaded. Connection requests from underloaded services should receive higher priority while connection requests from overloaded services should receive lower priority. The priority can be assigned following the $\mathrm{CP}$ admission control policy described in Section II. CP operates by using fixed values for the number of channels reserved for handoff requests, while VP adapts the number of channels reserved for handoff requests based on the expected nominal capacity (i.e., allocation) for each service.

\section{B. Policy Functions for VP}

VP changes the admission control parameters from $\mathrm{CP}$ (i.e., $T_{i_{s}}^{c}$ and $T_{k_{s}}^{w}$ ) based on the expected nominal capacities $C_{s}^{c}$ and $C_{s}^{w}$ for each service $s$, and the channel occupancies $\boldsymbol{m}_{\boldsymbol{i}}^{\boldsymbol{c}}$ and $\boldsymbol{m}_{\boldsymbol{k}}^{\boldsymbol{w}}$. We have: $T_{i_{s}}^{c}\left(\boldsymbol{m}_{\boldsymbol{i}}^{\boldsymbol{c}}\right)$ and $T_{k_{s}}^{w}\left(\boldsymbol{m}_{\boldsymbol{k}}^{\boldsymbol{w}}\right)$, which are functions of their respective channel occupancies. By following the notation for policy functions, we obtain for cell $i$ :

$$
\beta_{n_{i_{s}}}^{c}\left(\boldsymbol{m}_{\boldsymbol{i}}^{\boldsymbol{c}}\right)=\left\{\begin{array}{cc}
0, & \text { if } \sum_{s^{\prime} \in S} m_{i_{s^{\prime}}}^{c} b_{s^{\prime}} \leq T_{i_{s}}^{c}\left(\boldsymbol{m}_{\boldsymbol{i}}^{\boldsymbol{c}}\right) \\
1, & \text { otherwise }
\end{array}\right.
$$

and

$$
\beta_{h_{i_{s}}}^{c}\left(\boldsymbol{m}_{\boldsymbol{i}}^{\boldsymbol{c}}\right)=\left\{\begin{array}{cc}
0, & \text { if } \sum_{s^{\prime} \in S} m_{i_{s^{\prime}}}^{c} b_{s^{\prime}} \leq C_{i}^{c}-b_{s}, \\
1, & \text { otherwise }
\end{array}\right.
$$

where $T_{i_{s}}^{c}\left(\boldsymbol{m}_{\boldsymbol{i}}^{\boldsymbol{c}}\right)$ is given by

$$
T_{i_{s}}^{c}\left(\boldsymbol{m}_{\boldsymbol{i}}^{\boldsymbol{c}}\right)= \begin{cases}T H_{i_{s}}^{c}, & \text { if } m_{i_{s}}^{c} b_{s} \leq C_{s}^{c}-b_{s}, \\ T L_{i_{s}}^{c}, & \text { if } m_{i_{s}}^{c} b_{s}>C_{s}^{c}-b_{s},\end{cases}
$$

where $T H_{i_{s}}^{c}$ is the threshold value for high priority, and $T L_{i_{s}}^{c}$ for low priority. The priority in (9) is given by assigning the condition $T H_{i_{s}}^{c}>T L_{i_{s}}^{c}$. Note that setting $T H_{i_{s}}^{c}=T L_{i_{s}}^{c}$ for all $s \in S$, reduces VP to the $\mathrm{CP}$ admission policy. That is, the same parameter is used no matter if the service is underloaded or overloaded. Other forms for function $T_{i_{s}}^{c}\left(\boldsymbol{m}_{\boldsymbol{i}}^{\boldsymbol{c}}\right)$ can be considered. In the present work, we follow the form proposed in [5] based on the CP policy.

Similarly, for WLAN $k$, following the notation for policy functions we have:

$$
\beta_{n_{k_{s}}}^{w}\left(\boldsymbol{m}_{\boldsymbol{k}}^{\boldsymbol{w}}\right)=\left\{\begin{array}{cc}
0, & \text { if } \sum_{s^{\prime} \in S} m_{k_{s^{\prime}}}^{w} b_{s^{\prime}} \leq T_{k_{s}}^{w}\left(\boldsymbol{m}_{\boldsymbol{k}}^{\boldsymbol{w}}\right), \\
1, & \text { otherwise }
\end{array}\right.
$$

where $T_{k_{s}}^{w}\left(\boldsymbol{m}_{\boldsymbol{k}}^{\boldsymbol{w}}\right)$ is given by

$$
T_{k_{s}}^{w}\left(\boldsymbol{m}_{\boldsymbol{k}}^{\boldsymbol{w}}\right)= \begin{cases}T H_{k_{s}}^{w}, & \text { if } m_{k_{s}}^{w} b_{s} \leq C_{s}^{w}-b_{s} \\ T L_{k_{s}}^{w}, & \text { if } m_{k_{s}}^{w} b_{s}>C_{s}^{w}-b_{s}\end{cases}
$$

As in (9), the priority in (11) is given by assigning the condition $T H_{k_{s}}^{c}>T L_{k_{s}}^{c}$. In the case of policy function $\beta_{h_{k_{s}}}^{w}\left(\boldsymbol{m}_{\boldsymbol{k}}^{\boldsymbol{w}}\right)$, it is defined as (8) with the corresponding WLAN parameters (i.e., $m_{k_{s}}^{w}, C_{s}^{w}$ ).

\section{Optimal Admission Control}

To adjust the parameters of the policy functions $T H_{i_{s}}^{c}$, $T L_{i_{s}}^{c}, T H_{k_{s}}^{w}$ and $T L_{k_{s}}^{w}$, we propose the following blocking/dropping cost minimization problem. Minimize a linear objective function of the blocking and dropping probabilities for connection requests from new and handoff users:

$$
\begin{aligned}
\operatorname{minimize} \sum_{s \in S} \sum_{i \in M^{c}}\left[\alpha_{n_{i_{s}}}^{c} B_{n_{i_{s}}}^{c}+\alpha_{h_{i_{s}}}^{c} B_{h_{i_{s}}}^{c}+\right. \\
\left.\sum_{k \in W_{i}^{c}} \alpha_{n_{k_{s}}}^{w} B_{n_{k_{s}}}^{w}+\alpha_{h_{k_{s}}}^{w} B_{h_{k_{s}}}^{w}\right]
\end{aligned}
$$

$$
\begin{array}{ll}
\text { subject to } & B_{h_{i_{s}}}^{c} \leq \Gamma_{h_{i_{s}}}^{c}, \quad \forall i \in M^{c}, \quad \forall s \in S, \\
& B_{h_{k_{s}}}^{w} \leq \Gamma_{h_{k_{s}}}^{w}, \quad \forall k \in W_{i}^{c}, \forall s \in S, \\
& B_{n_{i_{s}}}^{c} \leq \Gamma_{n_{i_{s}}}^{c}, \quad \forall i \in M^{c}, \quad \forall s \in S, \\
& B_{n_{k_{s}}}^{w} \leq \Gamma_{n_{k_{s}}}^{w}, \quad \forall k \in W_{i}^{c}, \forall s \in S,
\end{array}
$$

where $\alpha_{n_{i_{s}}}^{c}$ and $\alpha_{n_{k_{s}}}^{w}$ denote the cost of blocking a connection request for service $s$ from a new user in cell $i$ and WLAN $k$, respectively. Similarly, $\alpha_{h_{i_{s}}}^{c}$ and $\alpha_{h_{k_{s}}}^{w}$ denote the cost of dropping a connection request for service $s$ from a handoff user in cell $i$ and WLAN $k$, respectively. To ensure that higher priority is considered for accepting connection requests from handoff users rather than new users, it is reasonable to set $\alpha_{n_{i_{s}}}^{c}<\alpha_{h_{i_{S}}}^{c}$ for all $i \in M^{c}$ and $s \in S$, and $\alpha_{n_{k_{s}}}^{w}<\alpha_{h_{k_{s}}}^{w}$ for all $k \in W_{i}^{c}$ and $s \in S . \Gamma_{h_{i_{s}}}^{c}$ and $\Gamma_{h_{k_{s}}}^{w}$ are the maximum dropping probabilities allowed for handoff connection requests, and $\Gamma_{n_{i_{s}}}^{c}$ and $\Gamma_{n_{k_{s}}}^{w}$ are the maximum blocking probabilities allowed for new connection requests in cell $i$ and WLAN $k$, respectively.

Note that the blocking and dropping probabilities $B_{n_{i_{s}}}^{c}$, $B_{h_{i_{s}}}^{c}, B_{n_{k_{s}}}^{w}$ and $B_{h_{k_{s}}}^{w}$ depend on the policy functions $\beta_{n_{i_{s}}}^{c}$, $\beta_{h_{i_{s}}}^{c}, \beta_{n_{k_{s}}}^{w}$ and $\beta_{h_{k_{s}}}^{w}$; whereas the policy functions depend on parameters $T H_{i_{s}}^{c}, T L_{i_{s}}^{c}, T H_{k_{s}}^{w}$ and $T L_{k_{s}}^{w}$. 
TABLE I

PARAMETERS OF THE NOMINAL ALLOCATIONS IN BBUS.

\begin{tabular}{|c|c|c|c|c|}
\hline Nominal Allocation & $C_{1}^{c}$ & $C_{2}^{c}$ & $C_{1}^{w}$ & $C_{2}^{w}$ \\
\hline \hline Type I & 5 & 26 & 15 & 44 \\
\hline Type II & 15 & 15 & 27 & 27 \\
\hline Type III & 26 & 5 & 44 & 15 \\
\hline
\end{tabular}

\section{NuMERICAL RESUltS AND Discussions}

An integrated cellular/WLAN system consisting of a cellular network with $\left|M^{c}\right|=3$ cells, and $\left|W_{i}^{c}\right|=2$ WLANs is considered. The cells and WLANs are enumerated as follows: $M^{c}=\{1,2,3\}, W_{1}^{c}=\{4,5\}, W_{2}^{c}=\{6,7\}$, and $W_{3}^{c}=$ $\{8,9\}$. Thus, inside the coverage of cell 1 , there are two WLANs given by WLAN 4 and WLAN 5 , respectively. In each cell $i \in M^{c}$ and in each WLAN $k \in W_{i}^{c}$, the network capacity is modeled as $C_{i}^{c}=30$ BBUs and $C_{k}^{w}=54$ BBUs, respectively. We assume that two different multimedia services are offered (i.e., $S=\{1,2\}$ ). The QoS provisioning requires that $b_{1}=1 \mathrm{BBU}$ and $b_{2}=2 \mathrm{BBU}$. In our study, we consider different traffic patterns by assigning different values to parameters $\lambda_{i_{s}}^{c}$ and $\lambda_{k_{s}}^{w}$, and assuming that $\lambda_{i_{s}}^{c}<$ $\lambda_{k_{s}}^{w}$ (i.e., WLANs are more densely populated [17]). For simplicity, we assume that connection requests from horizontal and vertical handoff users are equally important. We will investigate handoff differentiation in another submission.

We compare VP with three different settings for the nominal allocation, and CP policy [13]. The numerical values for the nominal allocation in cell $i$ for all $i \in M^{c}$ and WLAN $k$ for all $k \in W_{i}^{c}$ are shown in Table I. We select the values to show different performance of VP (i.e., different operation point). In nominal allocation type I, the allocation for service 2 is larger than for service 1 , while in type III, the nominal allocation for service 1 is larger than for service 2 . On the other hand, in nominal allocation type II, the allocation is the same amount in BBUs for both services, and set on half of the capacity of each network. Although the allocation is the same in BBUs, service 2 requires twice the resources for each connection.

For the admission control parameters, the blocking/dropping cost minimization problem (12) is solved by using an exhaustive search algorithm. For a larger problem, other more efficient meta-heuristic algorithms can be used [18]. Fig. 1 shows the minimum blocking/dropping cost values. Note that although the 3 nominal allocations for VP are close, VP type I achieves the lowest cost. VP type I is the nominal allocation with the largest $C_{2}^{c}$, then most of the time (i.e., channel occupancies $m_{1_{2}}^{c} b_{2}$ ), service 2 is considered underloaded and hence receiving high priority. We will discuss more on this issue when we analyze the blocking probabilities. In problem (12), we assume that, for all $i \in M^{c}, k \in W_{i}^{c}$, and $s \in S$, $\alpha_{n_{i_{s}}}^{c}=\alpha_{n_{k_{s}}}^{w}=1$ and $\alpha_{h_{i_{s}}}^{c}=\alpha_{h_{k_{s}}}^{w}=5$. That is, we assign five times higher priority to accept connection requests from handoff users compared to requests from new users. Blocking and dropping constraints are set $\Gamma_{h_{i_{1}}}^{c}=\Gamma_{h_{k_{1}}}^{w}=0.05, \Gamma_{h_{i_{2}}}^{c}=$ $\Gamma_{h_{k_{2}}}^{w}=0.1, \Gamma_{n_{i_{1}}}^{c}=\Gamma_{n_{k_{1}}}^{w}=0.1$, and $\Gamma_{n_{i_{2}}}^{c}=\Gamma_{n_{k_{2}}}^{w}=0.15$. From the results, the parameters for $\beta_{n_{i_{s}}}^{c}\left(\boldsymbol{m}_{\boldsymbol{i}}^{\boldsymbol{c}}\right)$ in (9) are set

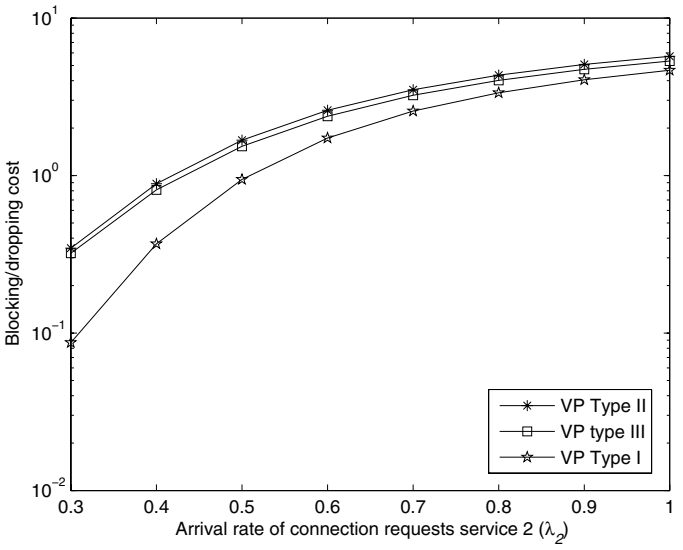

Fig. 1. Cost of blocking/dropping connection requests.

to $T H_{i_{s}}^{c}=27$ and $T L_{i_{s}}^{c}=24$ in cell $i$ for all $i \in M^{c}$. The admission control parameters for $\beta_{n_{k_{s}}}^{w}\left(\boldsymbol{m}_{\boldsymbol{k}}^{\boldsymbol{w}}\right)$ in (11) are set to $T H_{k_{s}}^{w}=50$ and $T L_{k_{s}}^{w}=44$ in WLAN $k$ for all $k \in W_{i}^{c}$. For CP policy $T_{i_{s}}^{c}=27$ and $T_{k_{s}}^{w}=50$. The values correspond to $\lambda_{2}=1$ new connection requests per minute. The optimization search and the fixed-point model of the integrated cellular/WLAN system are implemented in MATLAB.

\section{A. New Connection Blocking Probabilities}

Fig. 2 shows the probabilities of blocking connection requests from new users for both services in cell 1 and in WLAN 4. The arrival rate of connection requests from service 1 is 0.5 new connections per minute, while the arrival rate of service 2 is increased from 0.3 to 1 new connections per minute. In all figures, $\lambda_{s}=\lambda_{i_{s}}^{c}$ and $\lambda_{k_{s}}^{w}=\sigma \lambda_{i_{s}}^{c}$ new connection requests per minute. Since more traffic is generated in the WLANs, we assume that $\sigma=4$. Fig. 2(a) shows that in cell 1 for service 1 , as the number of connection requests increase the lowest blocking probabilities are archived by VP type III. Note that when $\lambda_{2}<0.5$, CP policy is slightly better, but as the number of connection requests increase VP adapts and outperforms CP. As service 2 overloads, less priority is given to its connection requests and hence protecting connection requests from service 1. On the other hand, Fig. 2(b) shows that for service 2 in cell 1 the lowest blocking probabilities are achieved by VP type I. As mentioned before, type I is the nominal allocation with the largest $C_{2}^{c}$, hence considering service 2 underloaded and with high priority. In fact, a large $C_{1}^{c}$ is the reason that VP type III achieves lower blocking probabilities for service 1 in Fig. 2(a). By giving service 2 in cell 1 high priority, its blocking probabilities decrease and hence the cost of blocking decrease as well, which is a dominant (i.e., large) term in the objective function of problem (12) as shown in Fig. 1. Additionally, Figs. 2(a) and 2(b) show that the worst nominal allocation for service 1 is VP type I, and for service 2 are VP types II and III, respectively. In both cases, it is because most of the time the services are considered overloaded (i.e., smaller $C_{1}^{c}$ and $C_{2}^{c}$ ), and hence with low priority. Fig. 2(c) shows that in WLAN 4 for service 1 the 3 allocation types of VP perform better than CP when $\lambda_{2}>0.5$, being the best VP types II and III. For 


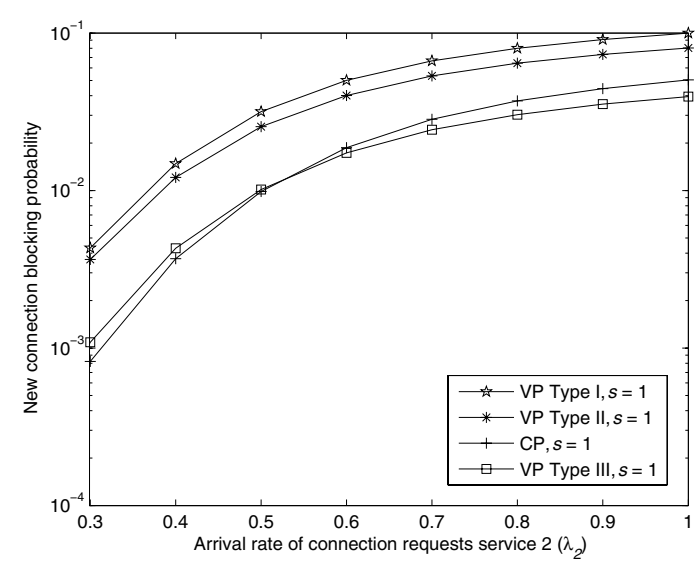

(a) cell $1, s=1$

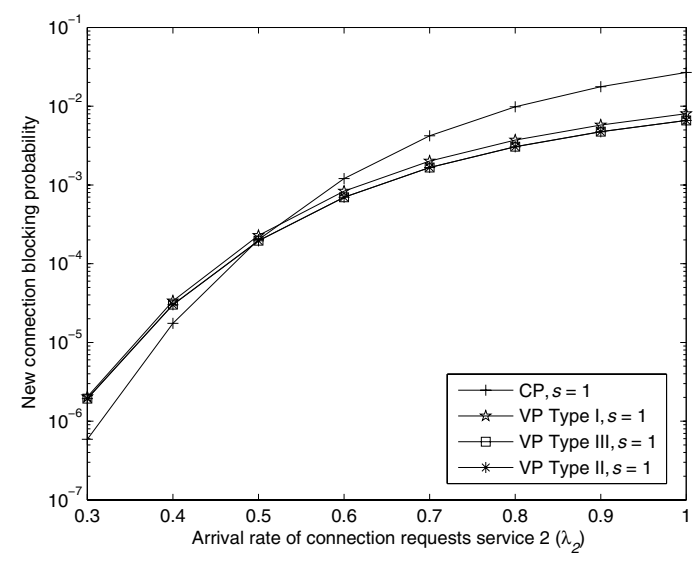

(c) WLAN $4, s=1$

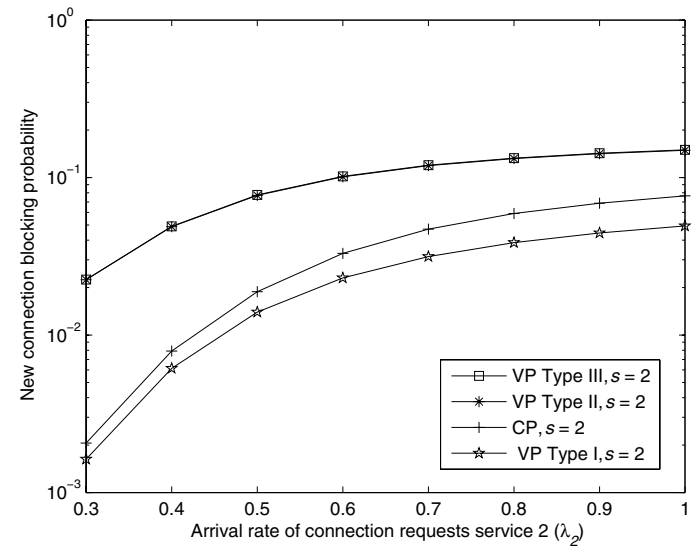

(b) cell $1, s=2$

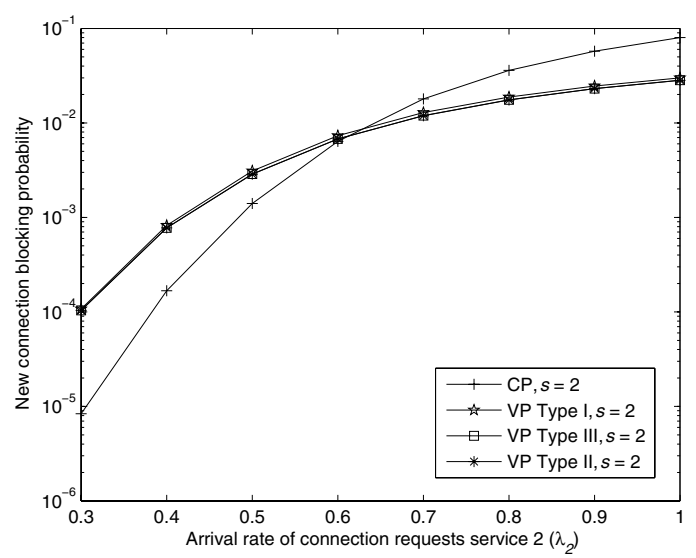

(d) WLAN $4, s=2$

Fig. 2. New connection requests blocking probabilities.

service 2 in WLAN 4, Fig. 2(d) shows that the 3 types of VP have lower blocking probabilities than CP when $\lambda_{2}>0.6$.

\section{B. Handoff Connection Dropping Probabilities}

Fig. 3 shows the probabilities of dropping connection requests from handoff users for both services in cell 1 and WLAN 4. The arrival rate of connection requests from service 1 is 0.5 , while the arrival rate of service 2 is increased from 0.3 to 1 new connections per minute. In Fig. 3(a), the lowest dropping probabilities for service 1 in cell 1 are achieved for VP type I. It is the nominal allocation that gives less priority to connections requests from the new users and hence favoring the requests from the handoff users. For service 2, Fig. 3(b) shows in cell 1 a similar behavior as service 1 , the 3 allocation types of VP achieve the lowest dropping probabilities, being the best VP types II and III. Finally, Figs. 3(c) and 3(d) show that in WLAN 4, for both services, the three types of nominal allocation for VP perform better than CP policy.

Although VP outperforms CP policy in a wide range of conditions due to its different assignment of priority based on the state of the service (i.e., overload/underload), the performance of VP for connection admission control is highly dependent on the nominal allocations (i.e., $C_{s}^{c}$ and $C_{s}^{w}$ ) which depend on the network capacities (i.e., $C_{i}^{c}$ and $C_{k}^{w}$ ). Thus, such values must be set based on the expected traffic for each service in each network, and considering the required QoS in terms of blocking and dropping probabilities.

\section{CONCLUSIONS}

In this paper we extended the virtual partitioning resource sharing scheme to improve connection admission control in cellular/WLAN interworking. Virtual partitioning is extended by using the concept of policy functions. The benefit of using virtual partitioning for admission control is evaluated by numerical results, and its performance is compared with the cutoff priority policy. It is shown that lower blocking and dropping probabilities for connection requests can be achieved by virtual partitioning in a wide range of conditions. For future work, we plan to consider other admission control policies and to include other types of wireless networks (e.g., WiMAX).

\section{ACKNOWLEDGMENT}

This work was supported by Bell Canada, the Natural Sciences and Engineering Research Council (NSERC) of Canada, and the Programa de Mejoramiento del Profesorado (PROMEP) from Mexico. 


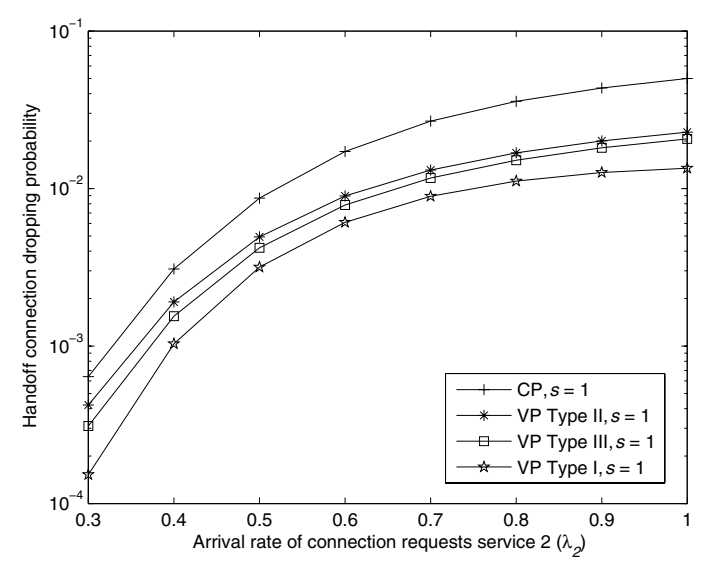

(a) cell $1, s=1$

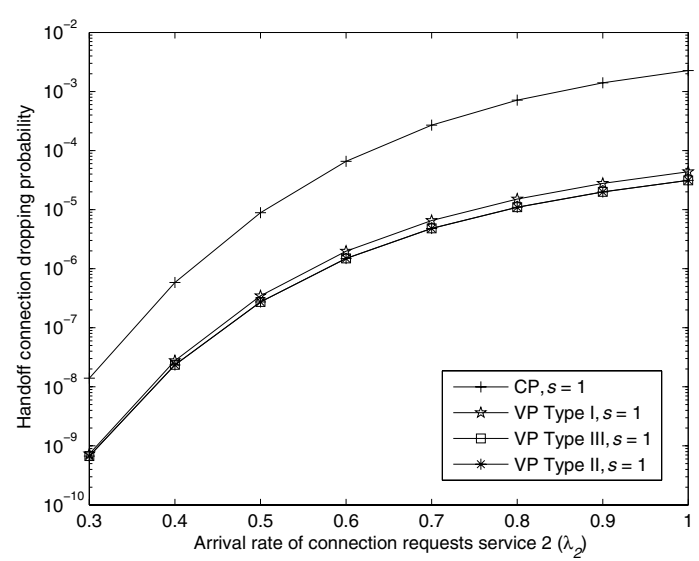

(c) WLAN $4, s=1$

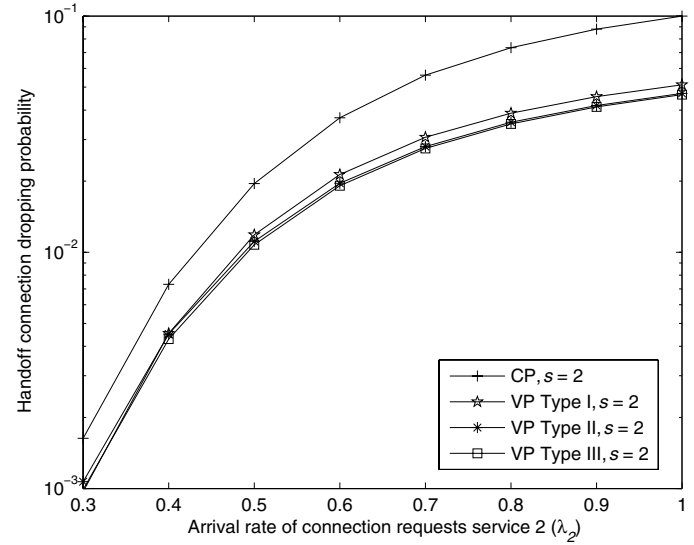

(b) cell $1, s=2$

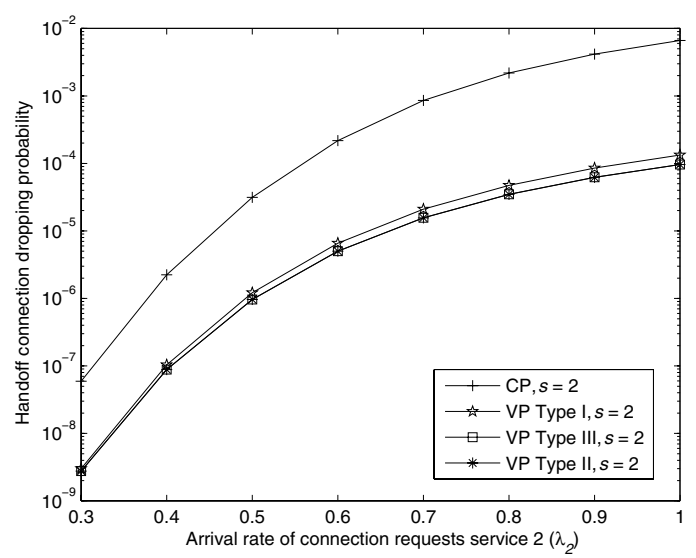

(d) WLAN $4, s=2$

Fig. 3. Handoff connection requests dropping probabilities.

\section{REFERENCES}

[1] A. Salkintzis, "Interworking Techniques and Architectures for WLAN/3G Integration Toward 4G Mobile Data Networks," IEEE Wireless Communications, vol. 11, no. 3, pp. 50-61, June 2004.

[2] IEEE 802.21 Working Group, http://www.ieee802.org/21/.

[3] 3GPP, "Requirements on 3GPP system to Wireless Local Area Network (WLAN) interworking," TS 22.234 (v8.0.0), March 2007.

[4] 3GPP2, "3GPP2-WLAN Interworking," S.R0087-A (v1.0), February 2006.

[5] S. Borst and D. Mitra, "Virtual Partitioning for Robust Resource Sharing: Computational Techniques for Heterogeneous Traffic," IEEE J. on Selected Areas in Communications, vol. 16, no. 5, pp. 668-678, June 1998.

[6] J. Yao, J. W. Mark, T. C. Wong, Y. H. Chew, K. M. Lye, and K. C. Chua, "Virtual Partitioning Resource Allocation for Multiclass Traffic in Cellular Systems With QoS Constraints," IEEE Transactions on Vehicular Technology, vol. 53, no. 3, pp. 847-864, May 2004.

[7] W. Song, H. Jiang, and W. Zhuang, "Performance Analysis of the WLAN-First Scheme in Cellular/WLAN Interworking," IEEE Transactions on Wireless Communications, vol. 6, no. 5, pp. 1932-1943, May 2007.

[8] W. Song, Y. Cheng, and W. Zhuang, "Improving Voice and Data Services in Cellular/WLAN Integrated Network by Admission Control," IEEE Transactions on Wireless Communications, in press, 2007.

[9] W. Xia and L. Shen, "Modeling and Analysis of Handoffs in Cellular and WLAN Integration," in Proc. of IEEE ICC'07, Glasgow, Scotland, June 2007.
[10] A. Hasib and O. Fapojuwo, "Performance Analysis of Common Radio Resource Management Scheme in Multi-service Heterogeneous Wireless Networks," in Proc. of IEEE WCNC'07, Hong Kong, China, March 2007.

[11] O. Sallent, J. Perez, C. Kloeck, I. Martoyo, S. Klett, and J. Luo, "Resource Auctioning Mechanism in Heterogeneous Wireless Networks," in Proc. of IEEE VTC'06 Spring, Melbourne, Australia, May 2006.

[12] E. Stevens-Navarro, A. H. Mohsenian-Rad, and V. W. S. Wong, "On Optimal Admission Control for Multi-Service Cellular/WLAN Interworking," in Proc. of IEEE GLOBECOM'07, Washington, DC, November 2007.

[13] Y. Fang and Y. Zhang, "Call Admission Control Schemes and Performance Analysis in Wireless Mobile Networks," IEEE Transactions on Vehicular Technology, vol. 51, no. 2, pp. 371-382, March 2002.

[14] R. Ramjee, D. Towsley, and R. Nagarajan, "On Optimal Call Admission Control in Cellular Networks," Wireless Networks, vol. 3, no. 1, pp. 29 41, March 1997.

[15] G. Bolch, S. Greiner, H. de Meer, and K. Trivedi, Queueing Networks and Markov Chains: Modeling and Performance Evaluation with Computer Science Applications. Wiley \& Sons, 1998.

[16] K. Ross, Multiservice Loss Models for Broadband Telecommunication Networks. Springer, 1995.

[17] A. Doufexi, E. Tameh, A. Nix, S. Armour, and A. Molina, "Hotspot Wireless LANs to Enhance the Performance of $3 \mathrm{G}$ and Beyond Cellular Networks," IEEE Communications Magazine, vol. 41, no. 7, pp. 58-65, July 2003.

[18] C. Blum and A. Roli, "Metaheuristics in Combinatorial Optimization: Overview and Conceptual Comparison," ACM Computing Surveys, vol. 35 , no. 3, pp. 268-308, 2003. 